\title{
MKS-Simulation eines Staplers mit aktivem Fahrwerk
}

\author{
DIPL.-ING. THORSTEN BIERMANN \\ EHEMALIGER WISSENSCHAFTLICHER ASSISTENT AM LEHRSTUHL FÜR MASCHINENELEMENTE UND TECHNISCHE LOGIS- \\ TIK DER HELMUT - SCHMIDT - UNIVERSITÄT HAMBURG
}

\section{Zusammenfassung}

In diesem Beitrag wird das Mehrkörpersystem-Modell (MKS-Modell) eines Gegengewichtsstaplers mit aktivem Fahrwerk erläutert. Die wesentlichen Merkmale des MKS-Modells sind die neuentwickelten Achsaufhängungen und die Hubgerüstlagerung des auf einen Gegengewichtsstapler mit 3 t - Tragkraft basierenden Fahrzeuges. Ein weiterer Aspekt ist die Kopplung der mechatronischen Modellteile Hydraulik und Regelung mit dem mechanischen Staplermodell. Abschließend zeigen die Ergebnisse der Co-Simulationen Verbesserungen der Fahrsicherheit und des Fahrkomforts.

\begin{abstract}
This report explains the multibody-system model (MBS) of a counterbalanced lift truck with active suspension. The main characteristics of the MBS model are the new developed suspensions and the fork-liftsuspension. The vehicle is based on a counterbalanced lift truck with a load capacity of 3 tonnes. A further aspect is the combined simulation (Co-simulation) of the mechatronic model hydraulic and control parts and the mechanical fork-lift-truck model. The results of the Co-simulation show an improvement in drivesafety and -comfort.
\end{abstract}

\section{Einleitung}

Steigende Anforderungen und Komplexität der Entwicklungsaufgaben, sowie erheblicher Zeit- und Kostendruck erfordern in vielen Bereichen des Maschinenbaus und der Fahrzeugindustrie den umfangreichen Einsatz von CAE-Werkzeugen zur effizienten Gestaltung des Entwicklungsprozesses. Insbesondere bei ganzheitlichen Entwicklungsansätzen, wie sie bei der Entwicklung mechatronischer Regelungssysteme Anwendung finden, sind geeignete virtuelle Modelle ein unverzichtbares Hilfsmittel, so auch bei der Entwicklung einer aktiven Federung für den Gegengewichtsstapler R 70-30 der STILL GmbH. In diesem Beitrag wird das Mehrkörpersystemmodell des Gegengewichtsstaplers mit aktiver Federung beschrieben, mit dessen Hilfe grundlegende Entwicklungsarbeiten und Funktionsnachweise des mechatronischen Systems durchgeführt wurden.

Mit dem Ziel die Fahrsicherheit, insbesondere die Kippstabilität, und den Fahrkomfort von Gabelstaplern signifikant zu verbessern, wurde ein aktives Fahrwerk für einen Gegengewichtsstapler entwickelt. Motivationen zur Entwicklung des Gabelstaplers mit aktivem Fahrwerk sind die Vermeidung von Unfällen, die Erhöhung der Kippstabilität, die Minderung der Schwingungsbelastung von Fahrzeug, Fahrer und Last, sowie die Erhöhung der Umschlagsleistung.

Zur Erreichung dieser Ziele bestand die Notwendigkeit sich vom herkömmlichen Fahrwerkskonzept bei Gabelstaplern zu trennen und eine völlige Neuentwicklung zu betreiben. Der Entwicklungsprozess eines solchen Systems umfasst zum einen Parameterstudien und zum anderen einen umfassenden Funktionsnachweis des Systems. Die Simulation mit wirklichkeitsnahen Modellen ist hierbei ein probates Hilfsmittel, die den Versuch in Teilen nicht nur kosten- und zeitgünstig ersetzt, sondern auch entsprechend ergänzt, beispielsweise durch Simulation von in experimentellen Untersuchungen nicht durchführbaren Szenarien oder durch die Bereitstellung nicht oder nur schwer messbarer Größen. Zur Untersuchung fahrdynamischer Fragestellungen eignet sich die Modellierung des Gegengewichtsstaplers mit aktivem Fahrwerk als Mehrkörpersystem aufgrund des benötigten Detaillierungsgrades und des Modellierungsaufwandes [Kortüm94], wobei die Ergänzung der numerischen Simulation sowohl durch theoretische Studien als auch durch Fahrversuche zur Verifizierung und Validierung des Simulationsmodells nötig ist. 


\section{Grundlagen des aktiven Fahrwerks und Fahrzeugkonzept}

Die Hauptaufgabe der Federung eines Fahrzeuges besteht im Allgemeinen in der Erhöhung des Fahrkomforts durch Minderung der Schwingungsbelastung von Fahrzeug, Fahrer und Last und der Verbesserung der Fahrsicherheit. Diese Forderungen führen bei der Auslegung einer Federung bereits auf einen Zielkonflikt zwischen Fahrsicherheit und Fahrkomfort, da hohe Fahrsicherheit durch eine „harte“ Federung und hoher Fahrkomfort durch eine „weiche“ Federung erreicht werden. Bei Gabelstaplern ist ferner die Kippstabilität bzw. Standsicherheit für die Fahr- und Betriebssicherheit entscheidend. Zur Lösung dieses Zielkonflikts sind das Aufbrechen der passiven Wirkungskette der Fahrzeugmassen und der Federung und das Einfügen aktiver Elemente notwendig. Aus der Fahrzeugtechnik ist eine Vielzahl aktiver Federungen bekannt, die als adaptive, semi-aktive, langsam aktive oder voll-aktive Systeme ausgeführt sind. Beim entwickelten Gabelstapler wurde eine langsame aktive Federung mit folgenden Eigenschaften umgesetzt:

- Rad- bzw. Achsaufhängungen

- $\quad$ Einfederung der Räder unabhängig voneinander

- Feder-Dämpfer-System

- Federsteifigkeit beladungsabhängig

- Einstellbare Dämpfungskonstante

- Aktive Stellfunktion

- $\quad$ Blockierung des Feder - Dämpfer - Systems

- $\quad$ Fahrwerksregelung

- $\quad$ Regelung der Aufbaubewegung

- $\quad$ Adaptive und aktive Eingriffe (minimaler Leistungsbedarf)

Beim Gabelstapler mit aktivem Fahrwerk handelt es sich um einen modifizierten Gegengewichtsstapler R 70 30 der STILL GmbH, dessen äußere Abmessungen und dessen Achslastverteilung gegenüber dem Serienfahrzeug unverändert blieben. Entsprechend den Anforderungen wurden die Vorderachse mit Hubgerüstlagerung, die Hinterachse, die Fahrwerkshydraulik und die Fahrwerksregelung entwickelt. Die Explosionsansicht der CADKonstruktion des Funktionsmusters ist in Abbildung 1 dargestellt.

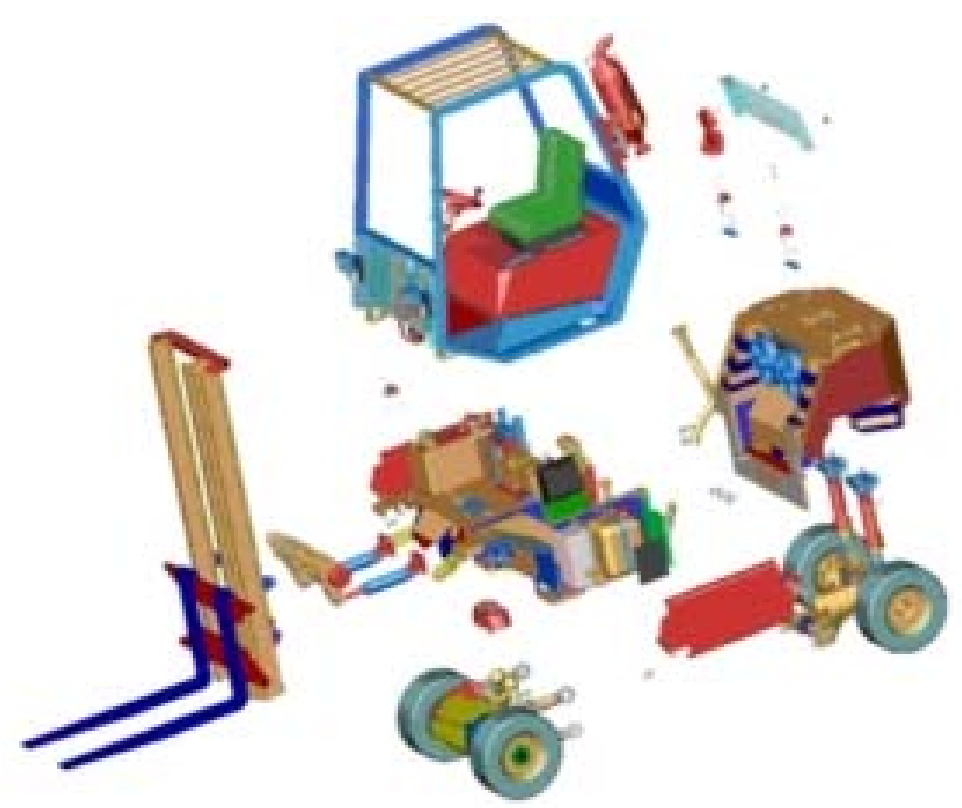

Abbildung 1: Explosionsansicht des Funktionsmusters 


\section{Modellbildung}

Im Folgenden wird das im Gesamtprojekt bereits zu einem relativ frühen Zeitpunkt entwickelte und eingesetzte Mehrkörpersystemmodell des aktiv gefederten Staplers beschrieben und erläutert. Das MKS-Modell beruht auf der Datenbasis des Referenzfahrzeuges STILL R 70-30 und enthält die neuentwickelten Achsaufhängungen und die Hubgerüstlagerung. Das Modell erlaubte noch vor Beginn der Konstruktionstätigkeiten die Untersuchung der Kinematik der beweglichen Bauteile inklusive Kollisionsprüfung. Ferner wurden Erkenntnisse zur geforderten Bauteilfestigkeit mittels spezieller Belastungsszenarien ermittelt. Die Modelle der Hydraulik und der Fahrwerksregelung wurden mit MATLAB/Simulink erstellt, sie wurden sowohl zur Systemauslegung als auch zum Funktionsnachweis benutzt.

Die Struktur des MKS-Modells wurde so gewählt, dass alle dynamischen Eigenschaften der Komponenten des Gabelstaplers, die die Fahrdynamik des Fahrzeuges im niederfrequenten Bereich beeinflussen, berücksichtigt wurden. Beim aktiv gefederten Stapler sind dies im Wesentlichen die Kinematik der Achsaufhängungen, des Hubgerüstes und der Lenkung, die elastischen Eigenschaften des Hubgerüstes, der Lagerung des Schutzdaches und des Antriebsaggregates sowie die dynamischen Eigenschaften der Reifen. Zudem sind noch einige nichtlineare Effekte, wie sie durch das Differenzialgetriebe beim Antrieb [Bruns03] und die Anschläge der Pendelachse verursacht werden, berücksichtigt. Das Gesamtmodell des Gegengewichtsstaplers zeigt die Abbildung 2.

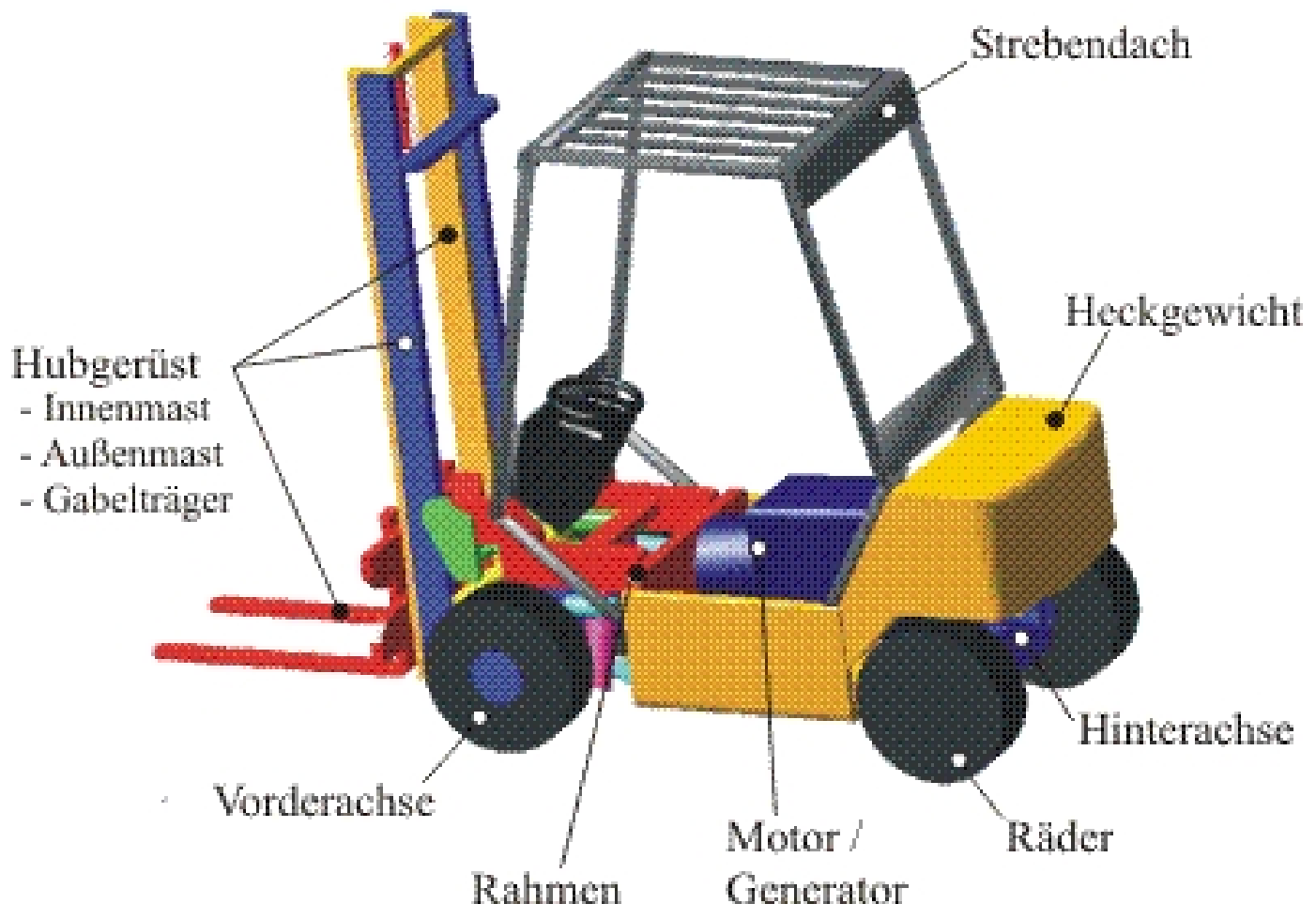

Abbildung 2: MKS-Modell des Gegengewichtsstaplers in ADAMS

Die Topologie des MKS-Modells ist in Abbildung 3 dargestellt, darin sind die Starrkörper als Ellipsen und die Gelenke als Kreise mit der entsprechenden Anzahl der jeweiligen Gelenkfreiheitsgrade dargestellt. Das MKSModell besteht aus $\mathrm{n}_{\mathrm{B}}=36$ Starrkörpern und $\mathrm{n}_{\mathrm{G}}=53$ Gelenken mit $\Sigma \mathrm{f}_{\mathrm{G}}=122$ Gelenkfreiheitsgraden, woraus sich nach dem Kutzbach-Kriterium die Zahl von $\mathrm{f}=20$ verallgemeinerten Freiheitsgraden und sieben kinematischen Schleifen für das MKS-Modell ergibt. Zur Modellierung beispielweise von Elastizitäten werden die Gelenke mit Kraftelementen überlagert. 


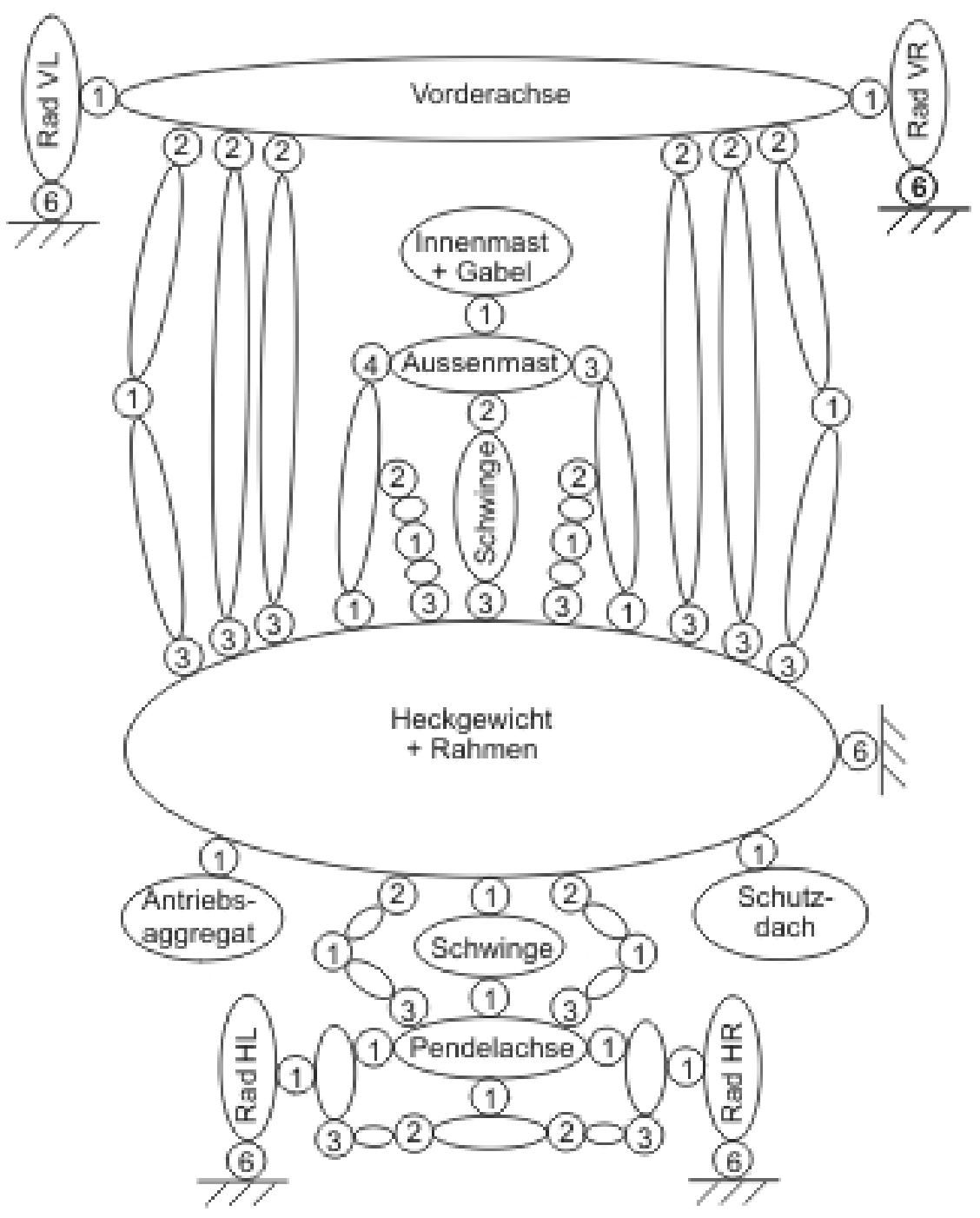

Abbildung 3: Topologie des MKS-Modell

\subsection{Vorderachse}

Die Vorderachse wurde als Vierlenker-Achsaufhängung entwickelt, die Einfeder- und Wankbewegungen der Achse und somit eine voneinander unabhängige Bewegung der Räder zulässt. Die übrigen Bewegungsmöglichkeiten der Achse wurden durch die Anordnung der Lenker unterbunden, wobei Seitwärtsbewegungen der Achse durch die diagonale Ausrichtung der beiden unteren Lenker verhindert werden.

Bei dieser Achsaufhängung konnte die serienmäßig verwendete Achse integriert werden, da die Entkopplung des elektrischen Antriebsstrangs zwischen dem am Motor angeflanschten Synchrongenerator und dem im Achskörper befindlichen Fahrmotor unproblematisch ist. Aus schwingungstechnischer Sicht musste jedoch der Nachteil des hohen Achsgewichts in Kauf genommen werden.

Aus Sicht der Modellbildung wurden sowohl die Lenker der Vorderachse als auch die Hydraulikzylinder beider Achsen jeweils mit einem Gelenkpaar Kugel - Kardangelenk gelagert, wodurch sogenannte isolierte Freiheitsgrade vermieden wurden. 


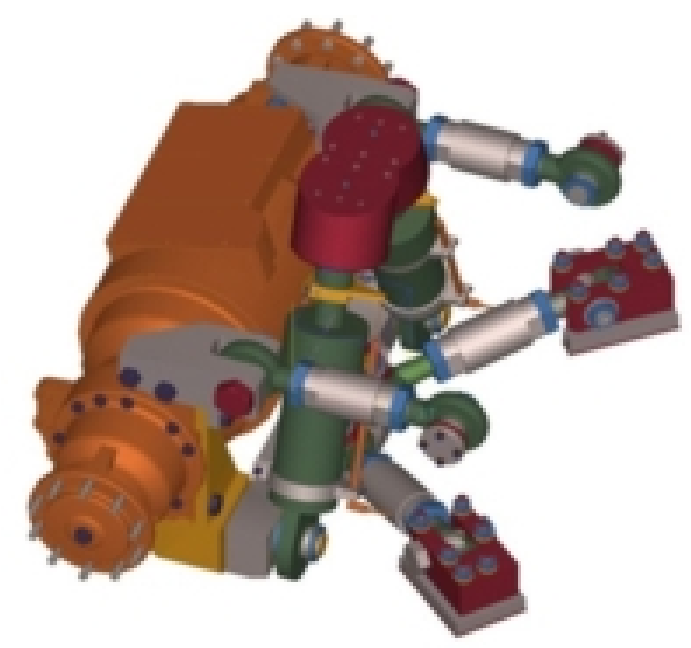

Abbildung 4: Vorderachse

\subsection{Hubgerüst/-lagerung}

Die Integration der Vorderachse ins Fahrzeug machte die Neuentwicklung der Hubgerüstlagerung notwendig, um bei Neigebewegungen eine Kollision mit der Vorderachse zu vermeiden. Zudem soll der Abstand zwischen Gabelrücken und Vorderachse möglichst gering sein. Dies konnte durch die Ausführung der Hubgerüstlagerung als Viergelenk-Kinematik, die eine gekoppelte Neige-/Hubbewegung ausführt, erreicht werden. Die Anbindung einer konventionellen Hubgerüstlagerung an die Achse ist sowohl aufgrund der kinematischen Gegebenheiten als auch aufgrund des Schwingungsverhaltens nicht sinnvoll.

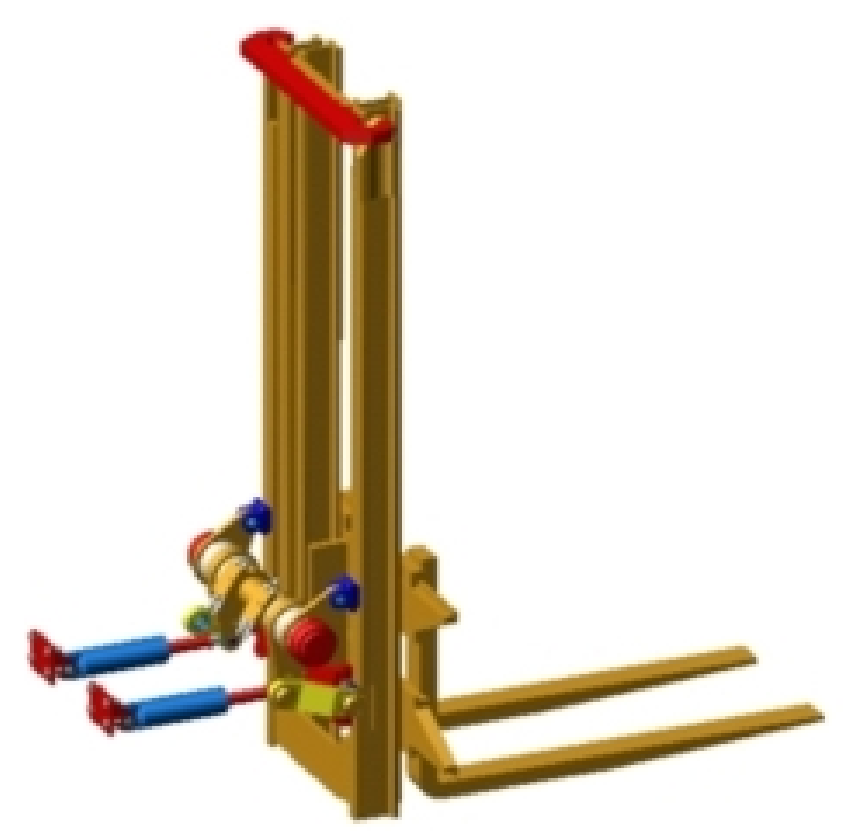

Abbildung 5: Hubgerüst/-lagerung

Für das relativ komplex aufgebaute Zweifachhubgerüst wurde eine Modellstruktur gewählt, mit der die wesentlichen elastischen Eigenschaften des Hubgerüstes vereinfacht modelliert werden, so dass der Einfluss der Hub-, Biegelängs- und Torsionschwingungen des Hubgerüstes, insbesondere bei beladenem Fahrzeug, in der Simulation berücksichtigt werden. Die dazu benötigten Ersatzsteifigkeiten werden nach der Theorie der Biegelinie 1. Ordnung approximiert und unter Berücksichtigung der Kinematik der Hubgerüstlagerung auf die Bewegung der Neigezylinder transformiert. Der Innenmast und die Gabel, gegebenenfalls mit Last, sind zu einem zweiten 
Starrkörper zusammengefasst, der mittels eines Schubgelenkes mit dem Außenmast verbunden ist. Das Schubgelenk ist wiederum mit einem Kraftelement überlagert, dass die Steifigkeiten der Kette und der Hydraulikzylinder in approximierter Form enthält.

\subsection{Hinterachse}

Die Lagerung der Pendelachse wurde um eine Schwinge erweitert, die um die Fahrzeugquerachse schwenkbar ist, so dass die Achse zwei Freiheitsgrade aufweist, wodurch wiederum eine in vertikaler Richtung voneinander unabhängige Bewegung der Räder ermöglicht wird. Der Achskörper wurde zur Lagerung der Federzylinder der Fahrwerksregelung und der Ausführung der Pendellagerung modifiziert. Die Ausführung der Lenkung entspricht der des Serienfahrzeuges.

Im Focus der Modellierung der Hinterachse liegt die stark nichtlineare Lenkkinematik, wobei Radträger, Spurstangen und Lenkzylinder zwar durch einfache Geometrien angenähert wurden, die für die Kinematik wesentlichen Längen und Abstände jedoch exakt eingehalten wurden. Die Lenkzylinderstellung kann sowohl intern als auch extern wahlweise mit einem Bewegungselement oder einem Kraftelement angesteuert werden. Auf diese Weise sind zum einem open- und closed-loop Fahrmanöver als Stand-alone-Simulationen durchführbar, zum anderen ist aber auch die externe Generierung von Lenkkräften, beispielsweise durch die Kopplung mit einem Control-System-Design-Tool (CSD-Tool), in dem die gesamte Lenkhydraulik modelliert ist, möglich.

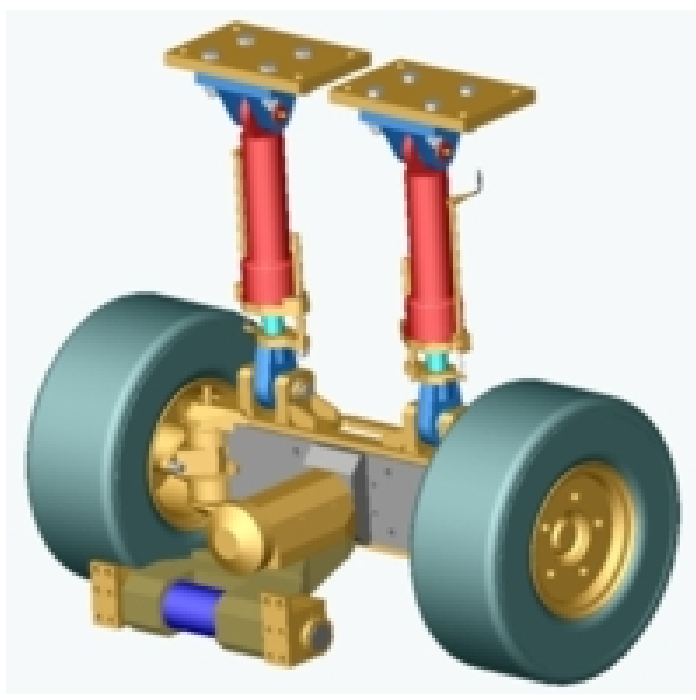

Abbildung 6: Hinterachse

\subsection{Motor/Strebendach}

Die elastisch gelagerten Komponenten Schutzdach und Antriebsaggregat sind in ihrer vertikalen Hauptschwingungsrichtung mittels Schubgelenken vom Fahrzeugaufbau entkoppelt. Die Schubgelenke sind mit Feder/Dämpferelementen, die die Steifigkeiten und Dämpfungen der Lagerungen enthalten, überlagert.

\subsection{Antrieb}

Das Fahrverhalten des Gabelstaplers in der Simulation hängt wesentlich von der Modellierung des mechanischen Differenzialgetriebes ab, da es in der Realität bei Kurvenfahrten durch das kurzzeitige Abheben des kurveninneren Antriebsrades, dem Stick-Slip-Effekt, zu Unterbrechungen der Antriebskraft kommt [Bruns03]. Das Antriebsmoment wird zunächst mittels eines P-Reglers in Abhängigkeit der Fahrgeschwindigkeit generiert, dies ist für quer- und vertikaldynamische Untersuchungen auch zulässig, zumal der zur Anregung des Modells genutzte Geschwindigkeitsverlauf durch entsprechende Messungen bereitgestellt oder synthetisch generiert wird. Die Einbindung eines detaillierteren Antriebsstrangmodells ist für quer- und vertikaldynamische Untersuchungen nicht notwendig, kann jedoch grundsätzlich erfolgen. Bei der symmetrischen Momentenaufteilung des Differenzialgetriebes auf die Antriebsräder ist jedoch der Zustand des Rad-Straße-Kontaktes zu berücksichtigen, da das Differenzialgetriebe aufgrund seines Laufgrades $f=2$ nur ein Moment gegen den ausreichenden Widerstand beider Räder übertragen kann. 


\subsection{Reifenmodell}

Wesentlichen Einfluss auf das Fahrverhalten des Simulationsmodells hat das verwendete Reifenmodell. Das MKS-Programm ADAMS stellt hierzu mehrere analytische Modelle zur Verfügung. Gemeinsam sind den Modellen die Algorithmen zur Modellierung der Kinematik des Radschlupfes und des Reibkontaktes des RadBoden-Kontaktes. Unterschiede hingegen bestehen bei den Formalismen zur Generierung der Reifenkräfte sowohl durch unterschiedliche Randbedingungen als auch durch den Umfang der Parametersätze. Für das Staplermodell wurde das UA-Tire-Model verwendet, wobei die zur Parametrierung benutzten Reifenkennlinien auf Prüfständen ermittelt wurden. Zur Validierung des gesamten Reifenmodells wurden Simulationen mit einem speziellen MKS-Modell zur Prüfung der Reifeneigenschaften durchgeführt und die Kennlinien der Seitenkraft und des Rückstellmomentes mit den Kennlinien aus den Versuchen abgeglichen.

\subsection{Fahrwerkshydraulik und -regelung}

Auf die Modelle der Fahrwerkhydraulik und -regelung wird nicht im Detail eingegangen. Die Fahrwerkshydraulik besteht im Wesentlichen aus Hydrospeichern als Federelemente, Proportionalventilen als aktive Stellglieder, Schaltventilen zur Einstellung der Dämpfung und den Hydraulikzylindern. Die Modelle beruhen auf den aus der Hydromechanik bekannten Gleichungen und wurden in MATLAB/Simulink unter Verwendung von $S$ functions programmiert. Ebenfalls in MATLAB/Simulink programmiert wurde die hierarchisch strukturierte Fahrwerksregelung, die mit leichten Schnittstellenmodifikationen mittels Rapid-Control-Prototyping im Funktionsmuster realisiert wurde.

Um ein im Sinne der Anforderungen optimales Betriebsverhalten zu erreichen, wurde eine hierarchisch aufgebaute Regelung entwickelt, deren Ziel zum einen die Horizontierung bzw. bei Kurvenfahrten die Überkompensierung der Wankbewegung des Fahrzeugaufbaus ist. Zudem ist der Fahrkomfort durch die Minimierung der Aufbaubeschleunigung zu erreichen.

\section{Die Co - Simulation}

Die Funktionsprüfung der Fahrwerksregelung erfolgte mittels Co-Simulation des Fahrzeugmodells in ADAMS und des Hydraulik- und Reglermodells in MATLAB/Simulink. Bei der hier durchgeführten Art der Co Simulation werden die Teilmodelle in ADAMS und in MATLAB/Simulink von eigenen Solvern gelöst. Die Ergebnisse der Solver werden mit fester Schrittweite übergeben, wodurch die maximale Schrittweite eines Solvers begrenzt ist. Die Übergabeschrittweite kleiner als das Fünffache des reziproken Wertes der maximalen Eigenfrequenz der Teilsysteme sein. Zu klein gewählte Schrittweiten erhöhen jedoch den Einfluss der Integrationsfehler.

An den Schnittstellen der Teilmodelle wurden spezielle Modifikationen durchgeführt, zwischen Fahrzeugmodell und Hydraulikmodell wurden die Bewegungsgrößen der Zylinder von ADAMS an MATLAB/Simulink übergeben und die Zylinderkräfte zurückgegeben. Ebenso wurden die zur Bestimmung des Fahrzeugzustandes benötigte Längs-, Quer- und Vertikalbeschleunigung und die Zylinderwege zur Regelung von ADAMS an MATLAB/Simulink übergeben. Die zur Ansteuerung der Proportionalventile benötigten Ströme berechnet der Regelalgorithmus und gibt sie MATLAB-intern an das Hydraulikmodell weiter. 


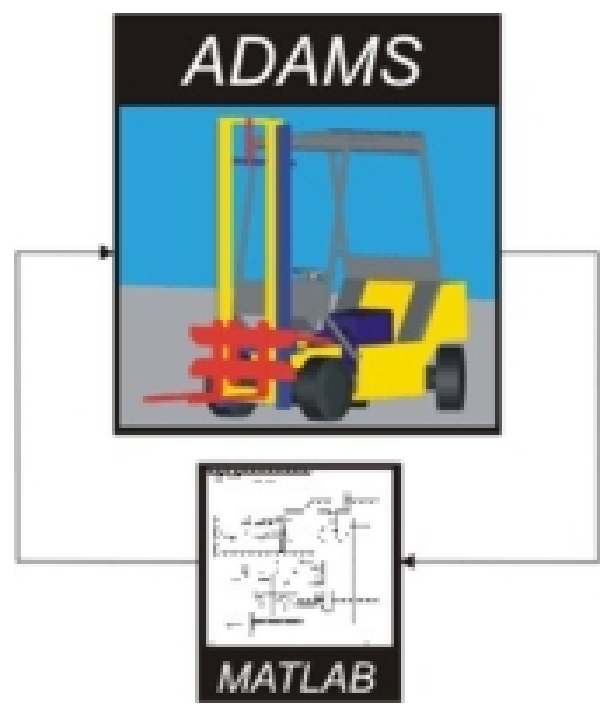

Abbildung 7: Co-Simulation

\section{Simulationsergebnisse}

Das Verbesserungspotenzial des Fahrkomforts und der Fahrsicherheit des Gegengewichtsstaplers mit aktivem Fahrwerk wurde mit zahlreichen Simulationen untersucht. Wesentliche Modellkomponenten, wie etwa das Reifenmodell, wurden vorab durch Fahrversuche mit einem ungefederten Stapler validiert.

Die Verbesserung des Fahrkomforts wird anhand der Simulation einer Schwellenüberfahrt gezeigt. Die Zeitverläufe und die entsprechenden Amplitudenspektren der Aufbauvertikalbeschleunigung sind in Abbildung 5 dargestellt. Der aktiv gefederte Stapler zeigt gegenüber dem ungefederten Stapler ein schnelles Abklingen der Aufbaubescheunigung und ein deutlich niedrigeres Amplitudenspektrum im Bereich niedriger Frequenzen bis $10 \mathrm{~Hz}$.
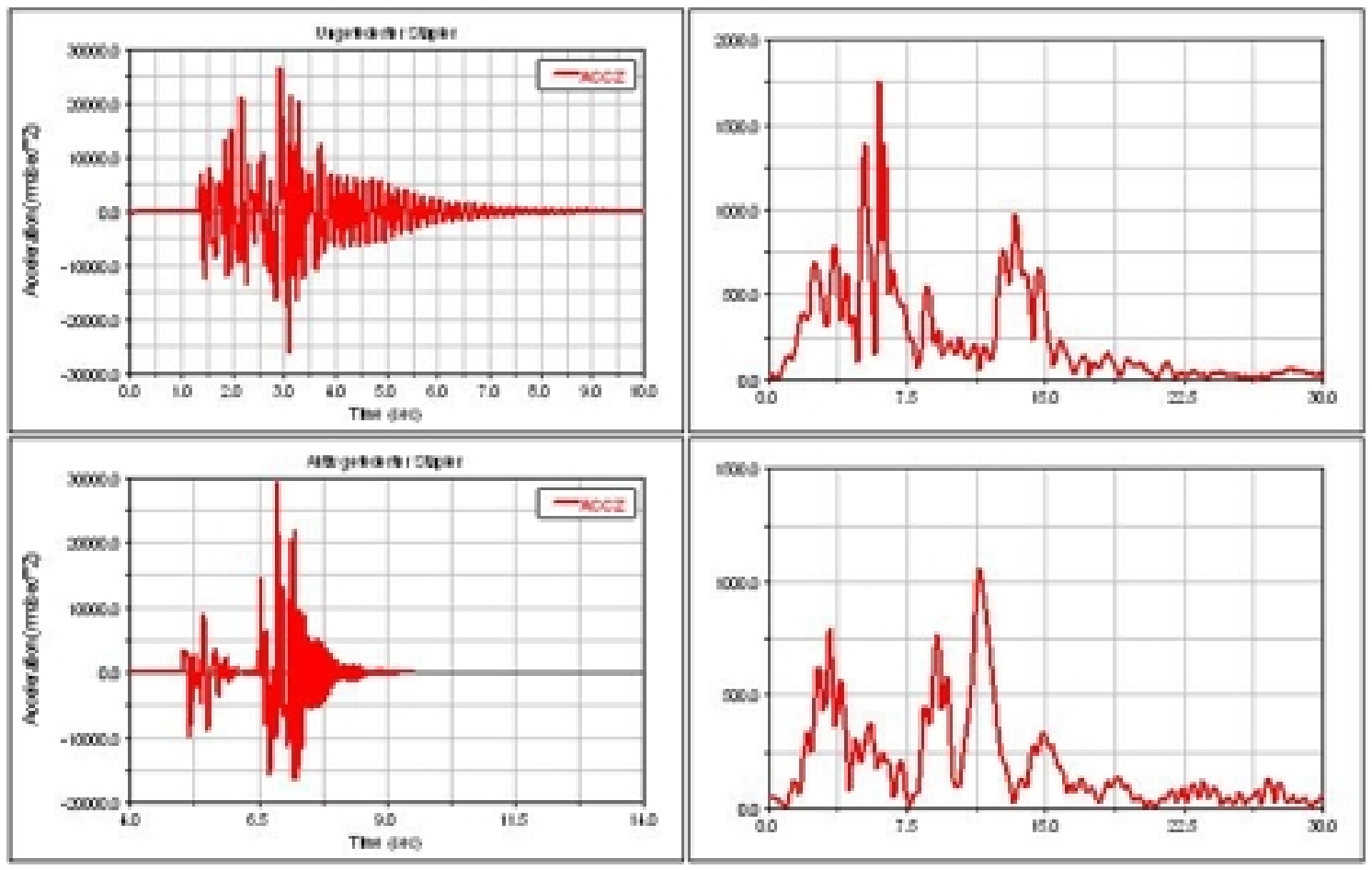

a) Aufbauvertikalbeschleunigung

b) Amplitudenspektrum

Abbildung 8: Aufbaubeschleunigung des ungefederten und aktiv gefederten Staplers 


\section{Zusammenfassung}

In diesem Beitrag wurde das MKS-Modell eines Gegengewichtsstaplers mit aktivem Fahrwerk erläutert. Die wesentlichen Merkmale des MKS-Modells sind die neuentwickelten Achsaufhängungen und die Hubgerüstlagerung des auf einen Gegengewichtsstapler mit 3 to - Tragkraft basierenden Fahrzeuges. Ein weiterer Aspekt war die Kopplung der mechatronischen Modellteile Hydraulik und Regelung mit dem mechanischen Staplermodell. Abschließend konnten die Ergebnisse der Co-Simulationen Verbesserungen der Fahrsicherheit und des Fahrkomforts zeigen.

\section{Literatur}

[Kortüm94]

Kortüm, W.; Lugner, P.; Systemdynamik und Regelung von Fahrzeugen, Berlin

[Bruns03] [u.a.]: Springer 1994. ISBN 3-540-57275-9

Bruns, R.: Fahrstabilität und Kippsicherheit von Gabelstaplern - Teil II, IN: F+H Fördern und Heben, 53 (2003) 1/2, S. 